\title{
Far-infrared-radio relation in cluster galaxies at intermediate redshift
}

\author{
Solohery M. Randriamampandry* \\ Astrophysics \& Cosmology Research Unit (ACRU), School of Maths, Statistics \& Computer \\ Science, University of KwaZulu-Natal, Durban 4041, South Africa \\ E-mail: soloherymampionona@gmail.com
}

\begin{abstract}
The radio luminosities at $1.4 \mathrm{GHz}$ is tightly correlated with the far-infrared luminosities for various galaxy types (e.g. [16, 6, 2]) over a wide range of redshift (see e.g. [5, 1, 15, 8, 7]). The relationship is widely believed to be driven by the internal star formation activity. Radio emission from these galaxies are predominantly produced from the synchrotron emission of cosmic-ray electrons accelerated in supernova shocks. The infrared emission is due to ultraviolet light from young massive stars that is absorbed and re-radiated by dust [3]. A correlation is found also in local clusters but cluster galaxies appears to have excess radio emission relative to the amount of far-infrared emission $[9,13,11]$. In this work, we measure the far-infrared-radio relationship in a massive cluster to test how this relationship changes at intermediate $\mathrm{z}$ between the field and a high-density cluster environment.
\end{abstract}

EXTRA-RADSUR2015 (*)

20-23 October 2015

Bologna, Italy

(*) This conference has been organized with the support of the Ministry of Foreign Affairs and International Cooperation, Directorate General for the Country Promotion (Bilateral Grant Agreement ZA14GR02 - Mapping the Universe on the Pathway to SKA)

\footnotetext{
* Speaker.
} 


\section{Motivation}

The main motivation in conducting this work consists of: (i) a number of work in the local Universe have reported that cluster galaxies appears to have excess radio emission relative to the amount of far-infrared emission $[9,13,11]$, (ii) so far, there is no investigations for the far-infraredradio relation in clusters at higher $\mathrm{z}$, (iii) thus this work aims to explore, for the first time, deviations or lack thereof of the far-infrared-radio relation in intermediate redshift cluster galaxies sample. In this contribution, we present results of studies performed for a massive galaxy cluster MS0451.60305 (hereafter, MS0451-03) at $\mathrm{z} \sim 0.538$.

\section{Observations \& Method}

The multi-wavelength data used in this work consists of: (i) optical spectroscopic redshift from Keck [4] and from the literature (an optical image of cluster MS0451-03 is displayed in Figure 1), (ii) VLA radio continuum observations at $1.4 \mathrm{GHz}$, and (iii) Spitzer MIPS and IRAC super mosaic imaging.

Radio data reduction and analysis were entirely carried out using the NRAO AIPS package. The $\mathrm{L}_{1.4 \mathrm{GHz}}$ were derived following the method of [10]. The IR photometry and data analysis were done using Spitzer MOPEX APEX software. The $\mathrm{L}_{\mathrm{IR}}$ were derived following the method of [14].

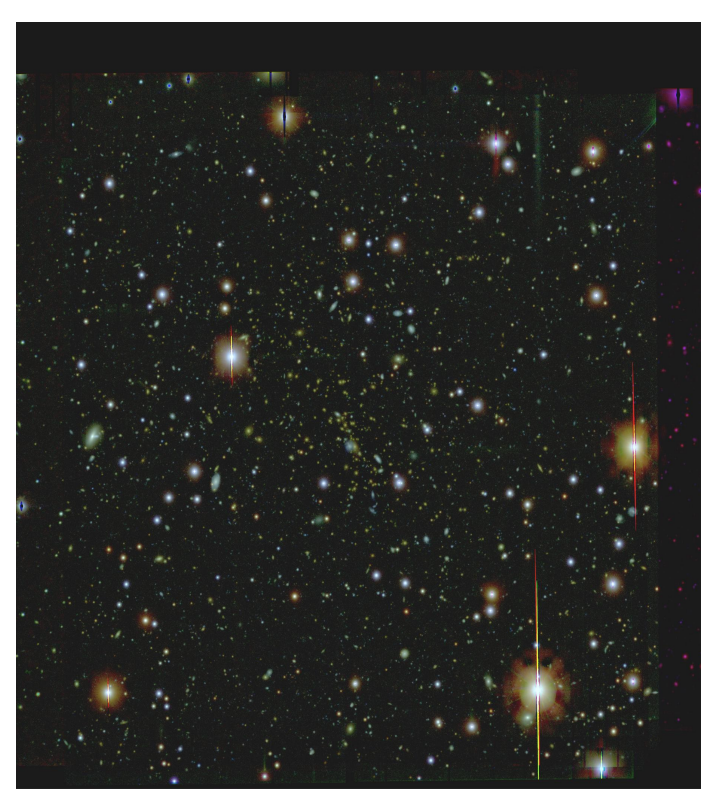

Figure 1: Galaxy Cluster MS 0451.6-0305 at $\mathrm{z}=0.538$.

\section{Results \& Summary}

We present our results in Figure 2 (for more details see [12]). The relationship between the 
rest frame radio luminosity at $1.4 \mathrm{GHz}\left(\mathrm{L}_{1.4 \mathrm{GHz}}\right)$ and the FIR luminosity $\left(\mathrm{L}_{60 \mu \mathrm{m}}\right)$ is shown. The solid line indicates the formal linear least-square fit of the cluster galaxies (see [13]) while the field relation (see [17]) is drawn using the dashed line.

In summary, we have constructed the far-infrared-radio relation for all sources with spectroscopic redshift using the Spitzer and VLA observations. We have found a correlation between the two parameters for the cluster star forming galaxies. We have measured $\mathrm{q}_{\mathrm{FIR}}$-value of $1.80 \pm 0.15$ with a dispersion of 0.53 for cluster galaxies which is in broad agreement, within uncertainties, with those for low z clusters (see [12]).

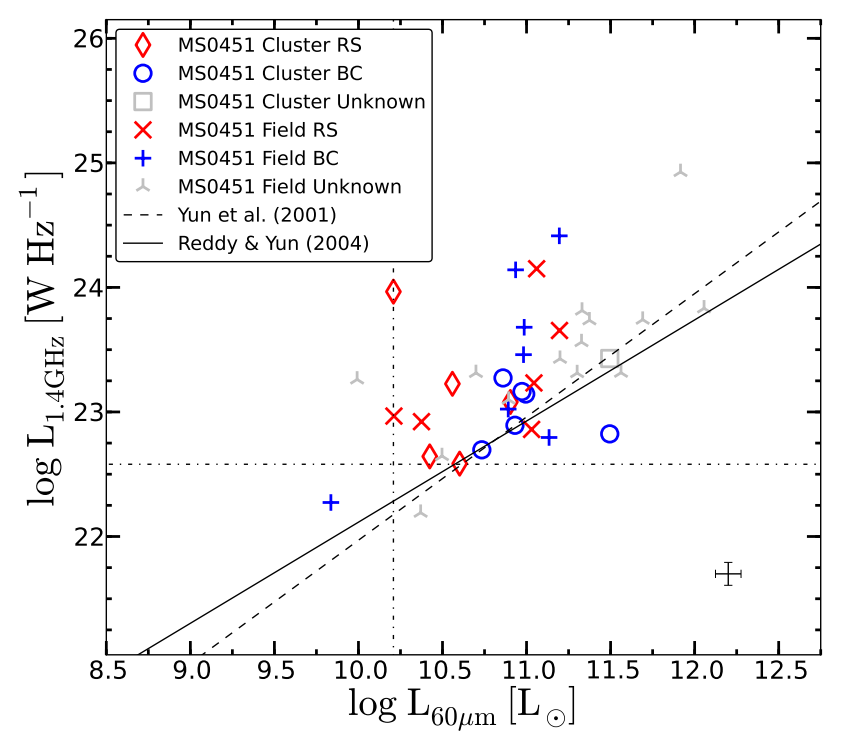

Figure 2: The rest frame radio luminosity at $1.4 \mathrm{GHz}\left(\mathrm{L}_{1.4 \mathrm{GHz}}\right)$ and the FIR luminosity $\left(\mathrm{L}_{60 \mu \mathrm{m}}\right)$ (see [12]).

\section{Future Work}

For further work, we: (i) shall expand the present sample into larger samples drawn from redMaPPer/ACT cluster sample in the SDSS Stripe 82 region and study the properties of star formation and AGN activity in clusters; (ii) shall use upcoming radio facilities such as MeerKAT/SKA which will enable us to further expand our sample to cover even more massive clusters at higher $\mathrm{z}$ to probe low luminosity star forming galaxies.

\section{Acknowledgments}

The financial assistance of the South African SKA Project (SKA SA) towards this research is hereby acknowledged. Opinions expressed and conclusions arrived at are those of the authors and are not necessarily to be attributed to the SKA SA (www.ska.ac.za). SMR acknowledges the support of the Ministry of Foreign Affairs and International Co-operation, Directorate General 
for the Country Promotion (Bilateral Grant Agreement ZA14GR02- Mapping the Universe on the Pathway to SKA)

\section{References}

[1] Appleton, P.N., Fadda, D.T., Marleau, F.R. et al. The Far- and Mid-Infrared/Radio Correlations in the Spitzer Extragalactic First Look Survey. ApJS, 154:147-150, September 2004.

[2] Condon, J.J., Anderson, M.L. and Helou, G. Correlations between the far-infrared, radio, and blue luminosities of spiral galaxies. ApJ, 376:95-103, July 1991.

[3] Condon, J.J. Radio emission from normal galaxies. ARA\&A, 30:575-611, 1992.

[4] Crawford, S.M., Wirth, G.D., Bershady, M.A. et al. Spectroscopy of Luminous Compact Blue Galaxies in Distant Clusters. I. Spectroscopic Data. ApJ, 741:98, November 2011.

[5] Garrett, M.A. The FIR/Radio correlation of high redshift galaxies in the region of the HDF-N. A\&A, 384:L19-L22, March 2002.

[6] Helou, G., Soifer, B.T. and Rowan-Robinson, M. Thermal infrared and nonthermal radio Remarkable correlation in disks of galaxies. ApJL, 298:L7-L11, November 1985.

[7] Ivison, R.J., Alexander, D.M., Biggs, A.D. et al. BLAST: the far-infrared/radio correlation in distant galaxies. MNRAS, 402:245-258, February 2010.

[8] Jarvis, M.J., Smith, D.J.B., Bonfield, D.G. et al. Herschel-ATLAS: the far-infrared-radio correlation at $z<0.5$. MNRAS, 409:92-101, November 2010.

[9] Miller, N.A. and Owen, F.N. The Far-Infrared-Radio Correlation in Nearby Abell Clusters. AJ, 121:1903-1914, April 2001.

[10] Morrison, G.E., Owen, F.N., Ledlow, M.J. et al. Radio-selected Galaxies in Very Rich Clusters at $z=$ 0.25. I. Multiwavelength Observations and Data Reduction Techniques. ApJS, 146:267-298, June 2003.

[11] Murphy, E.J., Kenney, J.D.P., Helou, G. et al. Environmental Effects in Clusters: Modified Far-Infrared-Radio Relations within Virgo Cluster Galaxies. ApJ, 694:1435-1451, April 2009.

[12] Randriamampandry, S. M., Crawford, S. M., Cress, C. M. et al. The far-infrared-radio correlation in MS0451-03. MNRAS, 447:168-177, February 2015.

[13] Reddy, N.A. and Yun, M.S. Radio and Far-Infrared Emission as Tracers of Star Formation and Active Galactic Nuclei in Nearby Cluster Galaxies. ApJ, 600:695-715, January 2004.

[14] Rieke, G.H., Alonso-Herrero, A., Weiner, B.J. et al. Determining Star Formation Rates for Infrared Galaxies. ApJ, 692:556-573, February 2009.

[15] Sargent, M.T., Schinnerer, E., Murphy, E. et al. The VLA-COSMOS Perspective on the Infrared-Radio Relation. I. New Constraints on Selection Biases and the Non-Evolution of the Infrared/Radio Properties of Star-Forming and Active Galactic Nucleus Galaxies at Intermediate and High Redshift. ApJS, 186:341-377, February 2010.

[16] van der Kruit, P.C. Observations of core sources in Seyfert and normal galaxies with the Westerbork synthesis radio telescope at 1415 MHz. A\&A, 15:110-122, November 1971.

[17] Yun, M.S., Reddy, N.A. and Condon, J.J. Radio Properties of Infrared-selected Galaxies in the IRAS 2 Jy Sample. ApJ, 554:803-822, June 2001. 